\title{
MARCEL BOURDETTE-DONON, Raymond Queneau, le Peintre de la vie moderne
}

\section{Riccardo Benedettini}

\section{(2) OpenEdition}

\section{Journals}

\section{Edizione digitale}

URL: https://journals.openedition.org/studifrancesi/12103

DOI: 10.4000/studifrancesi. 12103

ISSN: 2427-5856

\section{Editore}

Rosenberg \& Sellier

\section{Edizione cartacea}

Data di pubblicazione: 1 avril 2018

Paginazione: 165-166

ISSN: 0039-2944

\section{Notizia bibliografica digitale}

Riccardo Benedettini, «marcel bourdette-donon, Raymond Queneau, le Peintre de la vie moderne», Studi Francesi [Online], 184 (LXII | I) | 2018, online dal 03 juillet 2018, consultato il 17 novembre 2021. URL: http://journals.openedition.org/studifrancesi/12103; DOI: https://doi.org/10.4000/studifrancesi. 12103

Questo documento è stato generato automaticamente il 17 novembre 2021.

\section{(c) (i) (9)}

Studi Francesi è distribuita con Licenza Creative Commons Attribuzione - Non commerciale - Non opere derivate 4.0 Internazionale. 


\title{
MARCEL BOURDETTE-DONON, Raymond Queneau, le Peintre de la vie moderne
}

\author{
Riccardo Benedettini
}

\section{NOTIZIA}

MARCEl BoURDETTE-DONON, Raymond Queneau, le Peintre de la vie moderne, Paris,

L’Harmattan, 2016, «Espaces Littéraires», 317 pp.

1 Questo volume costituisce uno studio dell'opera di Raymond Queneau, scrittore, pensatore, matematico, figura di modello, di «classico», autore in grado di sorprendere e toccare il lettore di oggi, come Marcel Bourdette-Donon ricorda nella sua introduzione, «Le Peintre de la vie moderne» (pp. 7-18). Per illustrare come lo scrittore normanno, ma parigino d'adozione, abbia tentato di articolare le diverse sfere del sapere, l'A. sceglie di riflettere sui rapporti che i romanzi (ma in parte anche la poesia, la traduzione e i saggi) stabiliscono in una nuova percezione dello spazio-tempo, in un alternarsi continuo tra «l'urbanisation» e «le mouvement, [...] en symbiose avec la communication moderne» (p. 13). Bourdette-Donon conferma qui l'immagine di un Queneau estremamente colto che, sulla linea degli autori del Settecento, sa associare l'immaginazione e la fantasia dell'artista, la riflessione del filosofo in grado di mescolare il pensiero di Descartes, di Hegel e di Leibniz ai calcoli combinatori o alla memoria artificiale dei computer (p. 17). L'indagine si articola in tre capitoli «L'invention de nouveaux codes et de nouveaux modèles» (pp. 19-120), «L'évolution des représentations socioculturelles dans l'œuvre» (pp. 121-194) e «Les relations avec l'univers des normes» (pp. 195-304) - seguiti da un «Répertoire des auteurs, ouvrages, lettres et articles cités» (pp. 305-313) e da una rassegna di «Revues consacrées à Raymond Queneau» (p. 315).

2 L'A. dedica il primo capitolo del libro ad una descrizione dell'originalità letteraria dell'opera queniana, opera in cui lo scrittore ha saputo tener conto della dimensione ludica dello scambio mettendo alla prova i sistemi di comunicazione: sono analizzati i 
legami tra il tempo storico e il presente continuo, in particolare riferendosi ai rapporti conflittuali di Queneau con i viaggi (ricordiamo, in parallelo, un engagement nascosto dietro un apparente désengagement), soffermandosi sull'apertura letteraria all'Africa nera, ai problemi della decolonizzazione e all'interesse per la lingua negli anni in cui si cerca di uscire dalla guerra e si affrontano i totalitarismi. Rinviando a come questi testi si nutrano del linguaggio vivo di una comunità, dei suoi sogni e dei suoi miti, il secondo capitolo espone la visione della modernità secondo Queneau, i cui scritti spesso mettono in scena un'atmosfera "contrastée de féerie et de désespoir» (p. 123): l'antico e il nuovo sono messi a confronto con immagini del mondo contemporaneo (si pensi al cinema). Lo scrittore en situation, i cui romanzi riflettono un'epoca, le sue idee e le sue rappresentazioni, credenze e dottrine filosofiche, offre lo spunto al terzo capitolo, dedicato al tema dell'immaginazione sociale e culturale. Il volume mostra come con Queneau il romanzo abbia ritrovato il proprio carattere polimorfo, associando generi diversi per avvicinarsi e rispecchiare una nuova epoca, fatta di cambiamenti e sviluppi spesso violenti: Les temps mêlés, appunto. 